\title{
Philosophiques
}

\section{Bibliographie sélective sur la question des critères esthétiques}

Volume 23, numéro 1, printemps 1996

Critères esthétiques et métamorphoses du beau

URI : https://id.erudit.org/iderudit/027374ar

DOI : https://doi.org/10.7202/027374ar

Aller au sommaire du numéro

Éditeur(s)

Société de philosophie du Québec

ISSN

0316-2923 (imprimé)

1492-1391 (numérique)

Découvrir la revue

Citer ce document

(1996). Bibliographie sélective sur la question des critères esthétiques.

Philosophiques, 23(1), 133-137. https://doi.org/10.7202/027374ar d'utilisation que vous pouvez consulter en ligne.

https://apropos.erudit.org/fr/usagers/politique-dutilisation/ 


\section{BIBLIOGRAPHIE SÉLECIIVE SUR LA QUESTION DES CRIIËRES ESTHÉ IIQUES}

Beardly, Monroe, "What is an Aesthetic Quality?", The Aesthetic Point of View, Ithaca, Cornell U., 1982.

Aesthetics. Problems in the Philosophy of Criticism, New York, 1958.

Cauquelin, Anne. L'art contemporain, "Que sais-je ? ", $n^{\circ} 2671$, PUF, 1992.

"L'esthétique au risque des "Technimages" "dans $A$, Revue canadienne d'esthétique, tome I, 1996.

Chronis, Nikolaos, "The Evaluation of a Work of Art : Common Sense or Intersubjectivity? " (1984) dans Diotima, Athènes, 1986, $\mathrm{n}^{\circ} 4$, p. 101-107.

Collectif, The administration of Aesthetics (Censorship, Political Criticism, and the Public Sphere), Richard Burt, editor, "Cultural Politics ", vol. 7, Minneapolis, London, University of Minnesota Press, 1994.

Collectif, Théories esthétiques après Adorno (textes de H. R. Jauß, R. Bubner, K. H. Böhrer, P. Bürger, A. Wellmer), textes édités et présentés par Rainer Rochlitz, trad. fr. Rochlitz et Bouchindhomme, Actes Sud, 1990.

Collectif sous la dir. de Christian Descamps (J.-M. Schaeffer, J. Lacoste, F. Proust, C. Millot, F. Gaillard, C. Brunet, J,-P. Simon, O. Calabrese, D. Chateau), Le Beau aujourd'hui, Paris, "Espace international Philosophie ", Éditions du Centre Pompidou, 1993.

Collectif sous la dir. de C. Bouchindhomme et R. Rochlitz (T. De Duve, J.-M. Schaeffer, R. Shusterman, P. Bürger, C. Menke, M. Seel, A. Wellmer, C. Bouchindhomme et R. Rochlitz), L'art sans compas. Redéfinitions de l'esthétique. Collection "Procope ", Cerf, 1992.

Collectif, "L'art aujourd'hui " dans Esprit, juillet-aoūt 1991.

Collectif, "La crise de l'art contemporain " dans Esprit, février 1992.

Collectif, "L'art contemporain contre l'art moderne? " dans Esprit, octobre 1992.

Collectif, Philosophie analytique et esthétique, trad. et prés. D. Lories, Klincksieck, 1988.

Collectif, "La Beauté", Cahiers de l'Université, Paris, N. 16, "Convergences " $\mathrm{n}^{\circ} 5,1987, \mathrm{p} .9-207$.

Collectif dans la revue Spirale, $\mathrm{n}^{\circ} 138$, Dossier spécial *L'esthétique en éclats ", Montréal, décembre 1994-janvier 1995, p. 9-14. 
Danto, Arthur, L'Assujettissement philosophique de l'art, trad. fr. C. H. Schaeffer, Seuil, 1993.

De Duve, Thierry, "Cinq réflexions sur le jugement esthétique * (1993-1994) dans Du Nom au Nous, "Dis voir ", Octobre 1995. p. 45-69.

Dickie, George, Evaluating Art, Philadelphia, Temple University Press, 1988.

Dziemidok, Bohdan "On Aesthetic and Artistic Evaluations of the Work of Art * dans The Reasons of art/L'Art a ses raisons (P. J. McCormick ed.), University d'Ottawa Press, 1985, p. 295-306.

Gadamer, Hans-Georg, L'Actualité du beau, trad. fr. E. Poulain, Aixen-Provence, Alinea, 1992 (Die Aktualität des Schönen. Kunst als Spiel, Symbol und Fest, Stuttgart, Reclam, 1977.

Genette, Gérard, L'œuvre de l'art. Tome I : Immanence et transcendance, "Poètique ", Paris, Seuil, 1994.

Goldman, Alan, "Aesthetic Qualities and Aesthetic Value "dans Journal of Philosophy, 1990, 24.

Goodman, Nelson, "When is art?", Ways of Worldmaking, Indianapolis-Cambridge, Hackett Publishing Company, 1978 (trad. fr. dans Philosophie analytique et esthétique de D. Lories, Klincksieck, 1988).

Groys, Boris, Du nouveau. Essai d'économie culturelle, Chambon, 1995 (Carl Hanser Verlag, München, 1992).

Hauskeller, M. (Hrg.), Was das Schöne sei. Klassische Texte von Platon bis Adorno, Deutscher Taschenbuch Verlag, Wissenschaft 4626, 1994.

Jauß, H.R.(hrsg), Die nicht mehr schönen Künste, W. Fink Verlag, München, 1968.

Kamper, Dietmar und Christoph Wulf (hrsg), Der Schein des Schönen, Göttigen, 1989.

Kant, Immanuel, Critique de la faculté de juger, trad. fr. A. Philonenko, Paris, Vrin, 1965 (KantsWerke, Akademie Textausgabe V, Kritik der Urtheilskraft, Berlin, Walter de Gruyter \& Co., 1968).

Kieran, Matthew, "The Impoverishment of art ", The British Journal of Aesthetics, vol. 35, $\mathrm{n}^{\circ} 1$, January 1995.

Kupffer, H., "Was ist Kunst ? dans Universitas, 1988, 43, $\mathrm{n}^{\circ} 505$, p. 768-777.

Lorand, R., "Beauty - Order without Laws ", The Southern Journal of Philosophy 30 (1992), p. 42-63.

Lories, Danielle, Expérience esthétique et ontologie de l'œuvre, Académie royale de Belgique, 1989.

Luhmann, Niklas, Die Ausdifferenzierung des Kunstsystems, Bern, Benteli Verlag, 1994. 
Menke, Christoph, La souveraineté de l'art. L'expérience esthétique après Adorno et Derrida, trad. fr. Pierre Rusch, Paris, "Théories ", Armand Colin, 1993 (Die Souveränität der Kunst, Athenäum Verlag, 1988].

Moineau, Jean-Claude, "L'art sans art " dans Omnibus, Journal d'art trimestriel, $n^{\circ} 14$, octobre 1995.

Mothersill, Mary, Beauty Restored, Oxford, Clarendon Press, 1984.

Moulin, Raymonde, De la valeur de l'art (recueil), Flammarion, 1995.

Müller, Ulrich, "Wie ist ästhetisches Argumentieren möglich ? " dans Philosophisches Jahrbuch, vol. 96, 1, 1989, p. 151-156.

Osborne, Harold, "What is a work of art? dans British Journal of Aesthetics, vol. 21, 1981, p. 3-11.

Price, Kingsley, "What is a Piece of Music?" dans British Journal of Aesthetics, 22, Fall 82, p. 322-336.

Ramirez, Juan-Antonio, Les usines à valeurs. Écosystèmes des arts et explosion de l'histoire de l'art, Chambon, 1995 (Barcelone, Anagrama, 1994).

Rochlitz, Rainer, "L'identité de l'œuvre d'art "(sur Genette) dans Critique, $\mathrm{n}^{\circ}$ 574, mars 1995, p. 131-159.

Subversion et subvention. Art contemporain et argumentation esthétique, Paris, "NRF essais ", Gallimard, 1994.

"Critériologie du juste et du beau " dans Archives de philosophie $d u$ droit, tome 40: "Droit et esthétique", Paris, Sirey, 1995, p. 64-75.

"L'œuvre d'art et ses doubles "(sur Danto), dans Critique, n ${ }^{\circ} 514$, tome 46, mars 1990.

"Langage pour un, langage pour tous" (sur Seel) dans Critique $\mathrm{n}^{\circ}$ 488-489, tome 44, Jan-fév. 1988, p. 95-113.

Rosenkranz, Karl, Ästhetik des Häßlichen (1853), Hrsg. und mit einem Nachwort von Dieter Kliche, Leipzig, Reclam, 1990.

Rowe, M.W. "The definition of "Art" "dans Philosophical Quartely, 41 (164), July 1991, p. 271-286.

Ryan, Marie-Noëlle, "Guels critères, pour quelle critique ? " (sur Rochlitz) dans Spirale, $\mathrm{n}^{\circ}$ 138. Dossier spécial "L'esthétique en éclats ", Montréal, décembre 1994-janvier 1995, 10.

Schaeffer, J.-M. "L'œuvre d'art et son évaluation" dans Le Beau aujourd'hui, Paris, "Espace international Philosophie ", Éditions du Centre Pompidou 1993, p. 13-35. 
Seel, Martin, L'art de diviser. Le concept de rationalité esthétique, trad. fr. C.H.-Schaeffer, "Théories", Armand Colin, 1993 (Die Kunst der Entzweiung. Zum Begriff der ästhetischen Rationalität, Frankfurt am Main, Suhrkamp, 1985).

Eine Ästhetik der Natur, Suhrkamp, Frankfurt am Main, 1990.

"Was ist ein ästhetisches Argument?" dans Philosophisches Jahrbuch 94 (1987), p. 42 sqq.

* Die zwei Bedeutungen'kommunikative' Rationalität. Bemerkungen zu Habermas'Kritik der pluralen Vernunft "dans Kommunikatives Handeln. Beitrage zur J.H. "Theorie des kommunikativen Handelns * (A. Honneth $/ H$. Joas hrsg.), Frankfurt am Main, Suhrkamp, 1986.

"Le langage de l'art est muet" dans L'Art sans compas. Redéfinitions de l'esthétique, "Procope ", Cerf, 1992, p. 123-143.

Shusterman, Richard, "Lart en boite" (sur Danto), dans Critique, $n^{\circ}$ 562, mars 1994, p. 131-146.

L'art à l'état vif. La pensée pragmatiste et l'esthétique populaire, Paris, Minuit, 1991.

"L'Art et la théorie entre expérience et pratique "dans L'Art sans compas. Redéfinitions de l'esthétique, "Procope", Cerf, 1992, p. 45-76.

Soutif, Daniel, "La place du goūt dans la production philosophique des concepts et leur destin critique", dans Actes du colloque Archives de la critique d'art, 1992.

Stecker, Robert, "The End of an Institutional Definition of Art " dans The British Journal of Aesthetic, 26, Spr. 86, p. 124-132.

Steiner, George, Présences réelles. Les arts du sens, trad. fr., Gallimard, 1991.

Saint-Girons, Baldine, Fiat lux. Une philosophie du sublime, Paris, Vrin, 1995.

Swiderski, E. M.. "L'œuvre d'art en tant qu'objet esthétique. Complémentarité de perspectives sur une distinction problématique "dans Freiburger Zeitschrift für Philosophie und Theologie, $1986,33, \mathrm{n}^{\circ} 3$, p. 571-591.

Townsend, Dabney, Aesthetic Objects and Works of Art, Wolfeboro, Longwood, 1989.

Von Morstein, Petra, "Understanding Works of Art : universality, unity and uniqueness " dans British Joumal of Aesthetics 22, Fall 82 , p. 350-363.

Wohlfart, G., "Kunst oder Nicht-Kunst? Gedanken und Fragen zur Moderne "dans Wiener Jahrbuch für Philosophie, 1988, 20, p. 49-61. 
Yuktanandana, Aksak, "Musical Beauty and Levels of Hearing "dans The British Journal of Aesthetics, vol. 35, $\mathrm{n}^{\circ} 4$, october 1995, p. 49 sqq.

Zangwille, Nick, "The Beautiful, the Dainty and the Dumpy" dans The British Journal of Aesthetics, vol. 35, $\mathrm{n}^{\circ} 4$, october 1995 , p. 317-329. 